TRANSACTIONS OF THE

AMERICAN MATHEMATICAL SOCIETY

Volume 222, 1976

\title{
STABILITY THEOREMS IN SHAPE AND PRO-HOMOTOPY
}

BY

\author{
DAVID A. EDWARDS AND ROSS GEOGHEGAN $\left({ }^{1}\right)$
}

ABSTRACT. Conditions are given under which a topological space has the pointed shape of a $C W$ complex. These are derived from analogous conditions in pro-homotopy.

1. Introduction. A pointed connected topological space is stable if it is pointed shape equivalent to a pointed $C W$ complex. In [5] and [6] we gave necessary and sufficient conditions for a compact metric space (compactum) to be stable. In this paper, we generalize these criteria to arbitrary (pointed, connected) topological spaces. We also prove analogous theorems in pro-homotopy theory, but in this introduction we will only state the shape theorems.

Our first theorem (Theorem 3.2) says that a pointed connected space is stable if and only if it is pointed shape dominated by a pointed CW complex. It is an easy matter to deduce this from the compact case in [6]. The details are in $\S 3$.

Our second theorem (see Theorem 5.4 for a fuller version) says that $a$ pointed connected space whose strong shape dimension is finite is stable if and only if its homotopy pro-groups are dominated by groups. (Among the spaces with finite strong shape dimension are all finite-dimensional separable metric spaces: see §6.) Although the second theorem appears to be a generalization of the compact metric case treated in [5], the proof involves ideas which were not needed there. In the first place, we need the Bousfield-Kan spectral sequence [3]. Secondly, we need to know that if a pro-group $\left\{G_{\alpha}\right\}$ is pro-isomorphic to a group, then the derived limits $\lim ^{s}\left\{G_{\alpha}\right\}$ vanish for all $s \geqslant 1$ (if some of the groups $G_{\alpha}$ are nonabelian, only $\overleftarrow{\lim }^{1}\left\{G_{\alpha}\right\}$ is defined); in the abelian case, this latter result was announced by Verdier in [21]; we give a proof based on the Bousfield-Kan approach in $\S 4$. Thirdly, we need a Whitehead Theorem which is slightly different from that given in [5].

Received by the editors August 15, 1974.

AMS (MOS) subject classifications (1970). Primary 55D99; Secondary 14F99.

Key words and phrases. Pro-homotopy, pro-group, shape theory, derived limits, homotopy inverse limits.

(1) This work was completed while the second named author was visiting at the University of Georgia, whose hospitality he gratefully acknowledges. He was supported in part by NSF Grant PO 38761 . 
We thank Harold Hastings for helpful discussions.

2. Notation and terminology. If $I$ is a category, pro- $I$ is a category whose objects are inverse systems in $I$ indexed by directed sets. See [1] or [14] for a description of the morphisms of pro-I. We denote an object of pro-I by $\left\{X_{\alpha}\right\}_{\alpha \in A}$, or simply $\left\{X_{\alpha}\right\}$, where $\alpha$ ranges over some (variable) directed set $A$, $X_{\alpha}$ is an object of $I$, and, whenever $\alpha \leqslant \beta$, an unmarked morphism of $I$ from $X_{\beta}$ to $X_{\alpha}$ is understood to have been chosen in such a way as to make $\left\{X_{\alpha}\right\}$ an inverse system. These morphisms are called bonds. If $\alpha$ ranges over the set of natural numbers, $\left\{X_{\alpha}\right\}$ is called a tower.

We shall also need the category $I^{A}$ where $A$ is a directed set. Its objects are inverse systems in $I$ indexed by $A$. Its morphisms from $\left\{X_{\alpha}\right\}_{\alpha \in A}$ to $\left\{Y_{\alpha}\right\}_{\alpha \in A}$ are collections $\left\{f_{\alpha}: X_{\alpha} \rightarrow Y_{\alpha}\right\}_{\alpha \in A}$ of morphisms of $I$ which commute with the bonds.

An object $X$ of $I$ is dominated by an object $Y$ if there are morphisms

$$
X \underset{u}{\stackrel{d}{\rightleftarrows}} Y
$$

such that $d \circ u=1$, where 1 stands for the identity morphism.

$I_{\Delta}$ denotes the category whose objects are the commutative triangles in $I$, and whose morphisms are the commutative prisms in $I$.

The following categories appear: $T_{0}$ (pointed connected spaces and pointed maps); $C W_{0}$ (pointed connected $C W$ complexes and pointed maps); $H T_{0}, H_{0}$ (the pointed homotopy categories corresponding to $T_{0}$ and $C W_{0}$ ); $S S_{*}$ (pointed simplicial sets and pointed maps [17]); $K_{*}$ (pointed Kan complexes and pointed maps [17]); $H K_{*}$ (pointed $K a n$ complexes and pointed homotopy classes of pointed maps); $S S_{0}, K_{0}, H K_{0}$ (the full subcategories of $S S_{*}, K_{*}$ and $H K_{*}$ generated by connected objects); Groups (groups and homomorphisms); Abelian Groups (abelian groups and homomorphisms); Pointed Sets (pointed sets and pointed functions).

We always suppress base points when describing objects of these categories of pointed spaces. Similarly in the corresponding pro-categories. If $X=\left\{X_{\alpha}\right\}$ is an object of pro-CW $W_{0}$ or pro- $H_{0}, \pi_{k}(X)$ will denote the corresponding object $\left\{\pi_{k}\left(X_{\alpha}\right)\right\}$ of pro-Groups, where $\pi_{k}\left(X_{\alpha}\right)$ is the $k$ th homotopy group of $X_{\alpha}$. A morphism of pro-CW $W_{0}$ or pro- $H_{0}$ is a weak equivalence if it induces an isomorphism on each $\pi_{k}, k \geqslant 1$.

If $\left\{X_{\alpha}\right\}$ is in pro-CW $W_{0}$ we will usually also denote the induced object of pro- $H_{0}$ by $\left\{X_{\alpha}\right\}$.

$S: C W_{0} \rightarrow K_{0}$ and | $\cdot: K_{0} \rightarrow C W_{0}$ denote the singular-complex and geometric-realization functors [17]. 
The $C W$ dimension of a $C W$ complex $X_{\alpha}$ is the integer $C W-\operatorname{dim} X_{\alpha}$ such that the complex contains cells of that dimension, but of no higher dimension. If no such integer exists, $C W-\operatorname{dim} X_{\alpha}=\infty$. If $X=\left\{X_{\alpha}\right\}$ is an object of pro-CW, $C W-\operatorname{dim} X=\sup _{\alpha}\left\{C W-\operatorname{dim} X_{\alpha}\right\}$. The homotopy dimension of $X$ is $h-\operatorname{dim} X=$ $\inf \left\{C W-\operatorname{dim} Y \mid Y\right.$ is isomorphic to $X$ in pro- $\left.H_{0}\right\}$. The strong homotopy dimension of $X$ is $s-h-\operatorname{dim} X=\inf \{C W-\operatorname{dim} Y \mid Y$ is an object of pro-CW 0 which is isomorphic to $X$ in pro- $\left.H_{0}\right\}$.

An object $\left\{X_{\alpha}\right\}$ of pro-CW is compact if each $X_{\alpha}$ is a finite complex.

Our shape theory (pointed) is that of [13]; with very little change it could be that of [19]: the two agree on paracompact Hausdorff spaces [12], [18]. Thus it agrees with that of [8] on metric spaces [18], with that of [15] on compact Hausdorff spaces [13], and with that of [2] on compact metric spaces [16].

Following Morita [18], we say that an object $\left\{X_{\alpha}\right\}$ of pro- $H_{0}$ is associated with a pointed connected space $Z$ if (i) there are morphisms of $H_{0}, p_{\alpha}: Z \rightarrow$ $X_{\alpha}$ such that bond $\circ p_{\beta}=p_{\alpha}$ whenever $\alpha \leqslant \beta$; (ii) each morphism of $H_{0}, m: Z$ $\rightarrow P$ (where $P$ is an object of $H_{0}$ ) factorizes as $m=m_{\alpha} \circ p_{\alpha}$; and (iii) if $m_{\alpha} \circ p_{\alpha}=m_{\alpha}^{\prime} \circ p_{\alpha}$ are two factorizations, then there exists $\beta \geqslant \alpha$ such that $m_{\alpha} \circ$ bond $=m_{\alpha}^{\prime} \circ$ bond as morphisms of $H_{0}$ from $X_{\beta}$ to $P$.

Every pointed topological space $Z$ has a canonical object of pro- $H_{0}$ associated with it, namely the inverse system built from the nerves of all open locallyfinite normal (= numerable) covers of $Z$ exactly one of whose elements contains the base point $[18, \S 6]$.

Two objects of pro- $H_{0}$ are both associated with $Z$ if and only if they are isomorphic [18]. Two pointed spaces $Z$ and $Z^{\prime}$ are pointed shape equivalent if some (and hence any) object of pro- $H_{0}$ associated with $Z$ is isomorphic to an object associated with $Z^{\prime}$. $Z$ is pointed shape dominated by $Z^{\prime}$ if an object associated with $Z$ is dominated in pro- $H_{0}$ by an object associated with $Z^{\prime}$. Note, in this connection, that a pointed $C W$ complex is associated with itself.

We define pro- $\pi_{k}(Z)$ to be the pro-group $\left\{\pi_{k}\left(X_{\alpha}\right)\right\}$ where $\left\{X_{\alpha}\right\}$ is the canonical object of pro- $H_{0}$ associated with $Z$. Up to isomorphism in pro-Groups, any object associated with $Z$ would do as well.

Other terminology will be introduced as required.

3. Domination criteria for stability in pro-homotopy and shape. The following observation is elementary but important:

Lemma 3.1. Let $Y$ be an object of a category $I$, let $X$ be an object of pro-I and let 


$$
X \underset{u}{\stackrel{d}{\longrightarrow}} Y
$$

be morphisms of pro-I with $d \circ u=1_{X}$. Then $X$ is isomorphic in pro-I to the tower

$$
\{Y \stackrel{f}{\longleftarrow} Y \stackrel{f}{\longleftarrow} Y \stackrel{f}{\longleftarrow} \cdots\}
$$

where $f$ is the morphism of I defined by $u \circ d$.

PRoof. Routine. Compare with Proposition 3.1 of [6].

THEOREM 3.2. If an object $X$ of pro- $H_{0}$ is dominated in pro- $H_{0}$ by a pointed $C W$ complex $Y$, then $X$ is isomorphic in pro- $H_{0}$ to a pointed $\mathrm{CW}$ complex.

Proof. By Lemma 3.1 we may assume without loss of generality that $X$ is a tower $\left\{X_{n}\right\}$ in pro- $H_{0}$. By choosing representatives of the bonding homotopy classes, we get a tower in pro-CW $W_{0}$ which we also denote by $\left\{X_{n}\right\}$. The tower $\left\{S\left(X_{n}\right)\right\}$ in pro- $K_{0}$ is isomorphic in pro-HKo to a tower $\left\{Q_{n}\right\}$ of Kan fibrations (an object of pro- $K_{0}$ : compare Corollary 2.3 of [6]). Let $Q$ be the inverse limit of $\left\{Q_{n}\right\}$. Since the bonds are fibrations, $Q$ is clearly a Kan complex.

Let $p: Q \rightarrow\left\{Q_{n}\right\}$ be the canonical projection. For each $i \geqslant 0$ there is a short exact sequence (see $[3$, p. 254])

$$
* \rightarrow \underset{n}{\lim ^{1}} \pi_{i+1}\left(Q_{n}\right) \rightarrow \pi_{i}(Q) \stackrel{p_{\#}}{\longrightarrow} \stackrel{\lim }{\longleftarrow} \pi_{i}\left(Q_{n}\right) \rightarrow * .
$$

Since $\left\{Q_{n}\right\}$ is dominated in pro- $H K_{0}$ by a complex, all the $\lim ^{1}$ terms vanish. Hence $Q$ is connected and $p_{\#}$ is an isomorphism for $i \geqslant 1$.

There are morphisms

$$
\left\{Q_{n}\right\} \underset{u}{\stackrel{d}{\rightleftarrows}} S(Y)
$$

of pro- $H K_{0}$ such that $d \circ u$ is the identity. By the Covering Homotopy Property $\left[17\right.$, p. 30] $d$ is induced by a morphism of pro- $K_{0}$, which necessarily maps $S(Y)$ into the inverse limit, $Q$. Hence $d=p \circ d^{\prime}$ in pro-HKo, where $d^{\prime}: S(Y) \rightarrow Q$ is a map. Thus, in pro-HK,$p \circ\left(d^{\prime} \circ u\right)=1$, so $p$ has a right inverse. To see that $d^{\prime} \circ u$ is also a left inverse, note that

$$
\left(d^{\prime} \circ u\right)_{\#}: \underset{n}{\lim } \pi_{i}\left(Q_{n}\right) \rightarrow \pi_{i}(Q)
$$

is a right inverse for $p_{\#}$, hence a two-sided inverse ( $p_{\#}$ being an isomorphism). So $\left(d^{\prime} \circ u\right) \circ p: Q \rightarrow Q$ is a weak homotopy equivalence, hence a homotopy equivalence, hence the identity. Hence $X$ is isomorphic to $|Q|$. 
We will need the next proposition in $\$ 5$.

Proposition 3.3. If $\left\{G_{\alpha}\right\}$ is dominated in pro-Groups by a group $H$, then the projection $p: \lim \left\{G_{\alpha}\right\} \rightarrow\left\{G_{\alpha}\right\}$ is an isomorphism in pro-Groups.

Proof. Let

$$
\left\{G_{\alpha}\right\} \underset{u}{\stackrel{d}{\leftrightarrows}} H
$$

be morphisms of pro-Groups such that $d \circ u=1$. $d$ necessarily factorizes as $d$ $=p \circ d^{\prime}$ where $d^{\prime}: H \rightarrow \lim \left\{G_{\alpha}\right\}$. Thus $p$ has a right inverse $\left(d^{\prime} \circ u\right)$. It is easy to check that $\left(d^{\prime} \circ u\right) \circ p$ is an automorphism of $G$, and hence the identity.

We now use Theorem 3.2 to obtain a stability theorem in shape:

THEOREM 3.4. A pointed connected space $Z$ is pointed shape equivalent to a $C W$ complex if and only if $Z$ is pointed shape dominated by a $C W$ complex.

Proof. "Only if" is obvious. To prove "if" observe that an object of pro- $H_{0}$ associated with $Z$ will be dominated in pro- $H_{0}$ by a complex. Use Theorem 3.2. $\square$

For compact pro-complexes and spaces we can say a little more:

Theorem 3.5. Let $X$ be a compact object of pro- $H_{0}$. The following are equivalent:

(i) $X$ is dominated in pro- $H_{0}$ by a finite complex;

(ii) $X$ is isomorphic in pro- $H_{0}$ to a complex;

(iii) $X$ is dominated in pro- $H_{0}$ by a complex.

Proof. (ii) is equivalent to (iii) by Theorem 3.2. The proof that (i) is equivalent to (iii) is the same as the corresponding part of the proof of Theorem 1.1 of [6].

From this, we deduce

THEOREM 3.6. Let $Z$ be a pointed connected compact space. The following are equivalent (in pointed shape theory):

(i) $Z$ is shape dominated by a finite complex;

(ii) $Z$ is shape equivalent to a complex;

(iii) $Z$ is shape dominated by a complex.

REMARK 3.7. There remain the questions: when is a pro-complex isomorphic to a finite complex? and when is a space shape equivalent to a finite complex? By Theorems 3.5 and 3.6 we see that domination by a finite complex is necessary. But by Lemma 3.1 , domination by a finite complex implies 
isomorphism to a tower (or shape equivalence to a compact metric space). We have explained in 1.1 and 3.3 of [6], and in 4.2 of [5] that for finitely dominated towers (and compact metric spaces) the vanishing of a "Wall obstruction" is necessary and sufficient for isomorphism (or shape equivalence) to a finite complex; and all possible obstructions are realized. Thus, our questions are answered.

REMARK 3.8. There is also the question: when is a pro-complex isomorphic to a tower? A modification of Lemma 3.1 implies that an object of pro- $H_{0}$ is isomorphic to a tower if and only if it is dominated in pro- $H_{0}$ by a tower.

4. Homotopy limits and derived limits. If $\boldsymbol{A}$ is a directed set (or more generally a small category) Bousfield and Kan define a homotopy inverse limit functor holim ${ }_{A}:\left(S S_{*}\right)^{A} \rightarrow S S_{*}$ which associates a "best approximating" simpli-

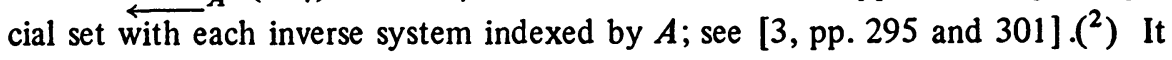
follows easily from Lemma 5.5 , p. 303, of [3] that the homotopy inverse limit of Kan complexes is a Kan complex, so we may write the restricted functoi as

$$
\operatorname{holim}_{A}:\left(K_{*}\right)^{A} \rightarrow K_{*}
$$

Let $i:\left(K_{*}\right)^{A} \rightarrow$ pro- $K_{*}$ and $p: K_{*} \rightarrow H K_{*}$ be the natural "inclusion" and "projection" functors. The principal theorem of this section is

THEOREM 4.1. There exists a functor holim: pro- $K_{*} \rightarrow H K_{*}$ such that for any directed set $A$, holim $\circ i=p \circ \operatorname{holim}_{A}$.

Proof. If $\left\{X_{\alpha}\right\}_{\alpha \in A}$ is an object of pro- $K_{*}$, define holim $\left\{X_{\alpha}\right\}$ to be $\operatorname{holim}_{A}\left\{X_{\alpha}\right\}$. Next, let $f:\left\{X_{\alpha}\right\}_{\alpha \in A} \rightarrow\left\{Y_{\beta}\right\}_{\beta \in B}$ be a morphism of pro-K $K_{*}$.

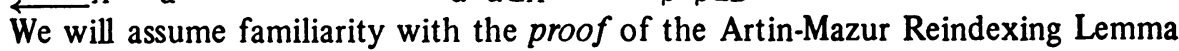
[1] as it appears in $\$ 2.2$ of [14]. From it we get a commutative diagram in pro- $K_{*}$

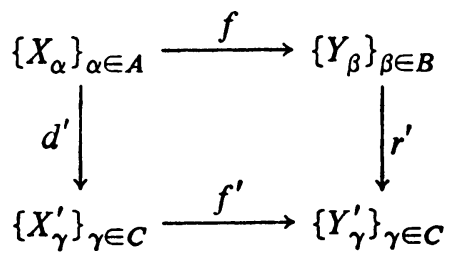

where $C$ is a directed set, $f^{\prime}$ is induced by a morphism $\left\{X_{\gamma}^{\prime} \stackrel{f_{\gamma}^{\prime}}{\longrightarrow} Y_{\gamma}^{\prime}\right\}$ of $\left(K_{*}\right)^{C}$,

(2) The homotopy inverse limit functor discussed explicitly in [3] is the unpointed version. However, as explained on p. 301 of [3], all the results we shall use from [3] have pointed analogues. See [7] for an alternative treatment of the material in Chapter XI of [3]. 
and $d^{\prime}$ and $r^{\prime}$ are induced by cofinal functors $d: C \rightarrow A$ and $r: C \rightarrow B$ (see [3, pp. 316-317]: we may regard a directed set as a small category). By the Cofinality Theorem [3, p. 317], $d^{\prime}$ and $r^{\prime}$ induce pointed homotopy equivalences $d_{*}: \operatorname{holim}_{A}\left\{X_{\alpha}\right\} \rightarrow \operatorname{holim}_{C}\left\{X_{\gamma}^{\prime}\right\}$ and $r_{*}: \operatorname{holim}_{B}\left\{Y_{\beta}\right\} \rightarrow \operatorname{holim}_{C}\left\{Y_{\gamma}^{\prime}\right\}$. There

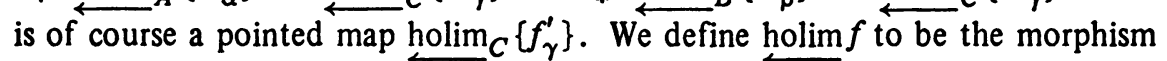
of $H K_{*}$ induced by $\left(r_{*}\right)^{-1} \longleftarrow_{\operatorname{holim}_{C}}\left\{f_{\gamma}^{\prime}\right\} \circ d_{*}$, where $\left(r_{*}\right)^{-1}$ is homotopy inverse to $r_{*}$.

We must now show that holim preserves identities and compositions.

Starting with $1_{\left\{X_{\alpha}\right\}}$, we $\overleftarrow{\text { have, }}$ as above, the commutative diagram

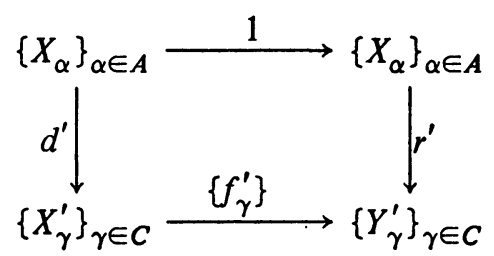

with $d^{\prime}$ and $r^{\prime}$ induced by cofinal functors $d, r: C \rightarrow A$. By referring to the definition of $C$ in $\$ 2.2$ of [14] , one sees at once that there is a cofinal "inclusion" functor $e: A \rightarrow C$ such that $d \circ e=r \circ e=1_{A}$. Furthermore, the definition implies that the following diagram commutes in pro- $K_{*}$

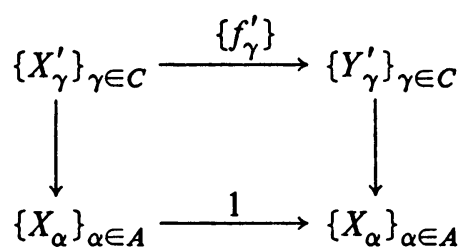

where the vertical morphisms are induced by $e$. The "naturality properties" of holim $_{A}$ and holim $C$ (see p. 296 of [3]) then give a commutative diagram in $K_{*}$

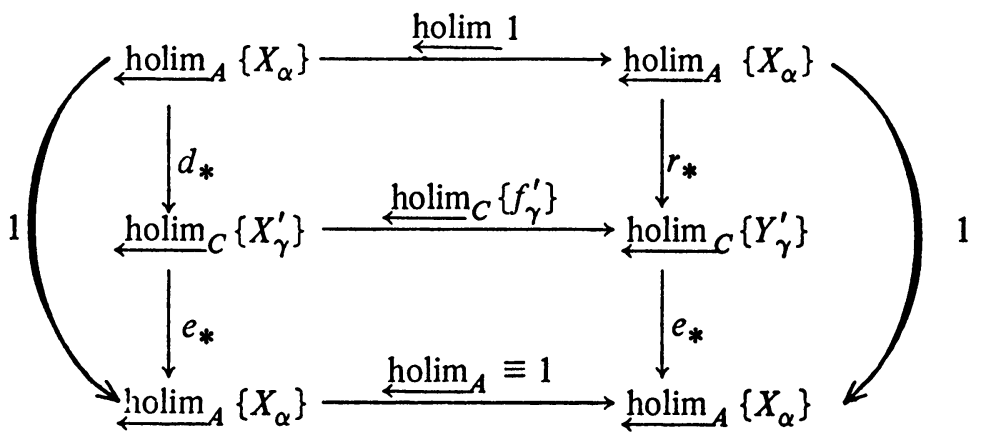

from which it follows that holim $1=1$ in $H K_{*}$.

The proof that holim preserves compositions uses the naturality properties in a similar way. We will give an outline which will enable the reader to construct the necessary diagrams and check that they commute. 
Let $h=g \circ f$ where $f:\left\{X_{\alpha}\right\}_{\alpha \in A} \rightarrow\left\{Y_{\beta}\right\}_{\beta \in B}$ and $g:\left\{Y_{\beta}\right\}_{\beta \in B} \rightarrow$ $\left\{Z_{\gamma}\right\}_{\gamma \in C}$ are morphisms of pro- $K_{*}$. Reindex $f_{;} g$ and $h$ as above to get $\left\{X_{\delta}^{\prime} \stackrel{f_{\delta}^{\prime}}{\longrightarrow} Y_{\delta}^{\prime}\right\}_{\delta \in D},\left\{Y_{\epsilon}^{\prime \prime} \stackrel{g_{\epsilon}^{\prime}}{\longrightarrow} Z_{\epsilon}^{\prime}\right\}_{\epsilon \in E}$ and $\left\{X_{\zeta}^{\prime \prime} \stackrel{h_{\zeta}^{\prime}}{\longrightarrow} Z_{\zeta}^{\prime \prime}\right\}_{\zeta \in F}$ where we have cofinal functors $d_{1}: D \rightarrow A, r_{1}: D \rightarrow B, d_{2}: E \rightarrow B, r_{2}: E \rightarrow C, d_{3}: F \rightarrow A$ and $r_{3}: F \rightarrow C$. As explained in $\$ 2.2$ of [14], $D$ is a directed set consisting of those morphisms $X_{\alpha} \rightarrow Y_{\beta}$ which can be "refined by" $f$, and the functors $d_{1}$ and $r_{1}$ pick out the domains and ranges. $E, d_{2}$ and $r_{2}$ are similarly related to $g$, as are $F, d_{3}$ and $r_{3}$ to $h$. Let $G$ be the directed set consisting of those compositions $X_{\alpha} \rightarrow Y_{\beta} \rightarrow Z_{\gamma}$ which can be "refined by" $h$, with the obvious partial ordering. There are obvious cofinal functors $m_{1}: G \rightarrow D, m_{2}: G \rightarrow E$ and $m_{3}: G \rightarrow F$, and we have $r_{1} m_{1}=d_{2} m_{2}, d_{3} m_{3}=d_{1} m_{1}$ and $r_{3} m_{3}=r_{2} m_{2}$. These equalities allow us to write

$$
(\text { holim } g) \circ\left(\operatorname{holim}_{\longleftarrow}^{\longleftarrow}\right)=\left(m_{2 *} r_{2}\right)^{-1} \operatorname{holim}_{G}\left\{g_{\eta}^{\prime \prime}\right\} \underset{\operatorname{holim}_{G}}{\longleftarrow}\left\{f_{\eta}^{\prime \prime}\right\} m_{1} * d_{1} *
$$

and

$$
\stackrel{\operatorname{holim}}{\longleftarrow}(g \circ f)=\left(m_{3^{*}} r_{3 *}\right)^{-1} \stackrel{\text { holim }}{\longleftarrow}\left\{g_{\eta}^{\prime \prime} f_{\eta}^{\prime \prime}\right\} m_{3^{*}} d_{3 *}
$$

A diagram similar to the one used in proving that holim preserves identities is used to show that holim ${ }^{\circ} i=p \circ$ holim $_{A}$. The argument contains no new ideas. $\square$

REMARK. Our pro- $K_{*}$ only contains inverse systems indexed by directed sets. But Theorem 4.1 also holds for the more general pro- $K_{*}$ defined in the Appendix to [1]; one must, of course, refer to pp. 160-162 of [1], rather than to [14] in the proof. (In fact for any category $I$, pro-I using directed sets is equivalent to pro-I using filtered categories: see [7].)

If $\left\{G_{\alpha}\right\}$ is an object of pro-(Abelian Groups) there exists, for each integer $s \geqslant 0$, the derived limit abelian group $\lim ^{s}\left\{G_{\alpha}\right\}$ : see $[3$, p. 305], for the definition and references; $\lim ^{0}\left\{G_{\alpha}\right\}$ is the ordinary inverse limit abelian group. If $\left\{G_{\alpha}\right\}$ is an object of pro-Groups, the derived limits $\lim ^{0}\left\{G_{\alpha}\right\}$ and $\lim ^{1}\left\{G_{\alpha}\right\}$ are introduced in [3, p. 307]; in this latter case, $\lim ^{0}\left\{G_{\alpha}\right\}$ is the ordinary inverse limit group, and $\lim ^{1}\left\{G_{\alpha}\right\}$ is a pointed set.

CoRollary 4.2. (i) If $\left\{G_{\alpha}\right\}$ is isomorphic in pro-(Abelian Groups) to an abelian group $G$, then $\lim ^{s}\left\{G_{\alpha}\right\}$ is trivial for all $s \geqslant 1$.

(ii) If $\left\{G_{\alpha}\right\}$ is isomorphic in pro-Groups to a group $G$, then $\lim ^{1}\left\{G_{\alpha}\right\}$ is trivial.

PROOF. We prove (i): (ii) is proved similarly. For $n \geqslant 1$ we have the Eilenberg-Mac Lane functor $K(\cdot, n)$ : (Abelian Groups) $\rightarrow K_{*}$ : see $[17$, pp. 88 
and 98-100]; this functor automatically extends to pro-categories. Using Theorem 4.1, we have $\pi_{1} \operatorname{holim}\left\{K\left(G_{\alpha}, s+1\right)\right\}$ isomorphic to $\pi_{1} \underset{h o l i m}{\longleftarrow} K(G, s+1)$. The first of these groups is isomorphic to $\lim ^{s}\left\{G_{\alpha}\right\}$ while the second is isomorphic to $\lim ^{s} G$ : see [3, p. 309]. Here $G$ stands for an inverse system indexed by a one-element directed set; for such a system $\lim ^{s}$ vanishes when $s \geqslant 1$ : see $\left[3\right.$, p. 306]. So $\lim ^{s}\left\{G_{\alpha}\right\}$ vanishes.

5. Algebraic criteria for stability in pro-homotopy. If $\left\{Y_{\alpha}\right\}_{\alpha \in A}$ is an object of $\left(K_{*}\right)^{A}$, there are canonical maps $p_{\alpha_{0}}: \operatorname{holim}_{A}\left\{Y_{\alpha}\right\} \rightarrow Y_{\alpha_{0}}$ for each $\alpha_{0}$ $\in A$, such that $p_{\alpha_{0}}$ is pointedly homotopic to bond $\circ p_{\alpha_{1}}$; see Proposition 3.4, p. 296 of [3]. These define a morphism $p: \operatorname{holim}\left\{Y_{\alpha}\right\} \rightarrow\left\{Y_{\alpha}\right\}$ in pro-HK *. In this section we will discuss conditions which make $p$ an isomorphism.

Lemma 5.1. Assume each $Y_{\alpha}$ is connected. Then holim $\left\{Y_{\alpha}\right\}$ is connected and $p$ is a weak equivalence if and only if $\left\{\pi_{i}\left(Y_{\alpha}\right)\right\}$ is dominated in pro-Groups by a group, $i \geqslant 1$.

Proof. "Only if" is obvious. In fact, by Proposition 3.3, we can conclude that $\left\{\pi_{i}\left(Y_{\alpha}\right)\right\}$ is isomorphic to $\lim \left\{\pi_{i}\left(Y_{\alpha}\right)\right\}$. We prove "if". The following diagram commutes (in pro-Groups $\overleftarrow{\text { if }} i \geqslant 1$, in pro-(Pointed Sets) if $i=0$ ):

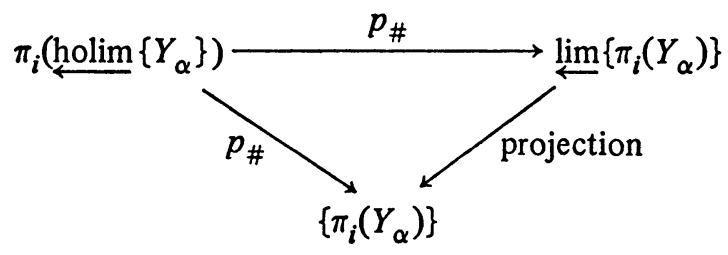

By Proposition 3.3, "projection" is an isomorphism if $i \geqslant 1$; and "projection" is trivially an isomorphism if $i=0$. Hence it will be enough to show that the horizontal $p_{\#}$ is an isomorphism. That this is so follows from the convergence of the Bousfield-Kan spectral sequence [3, p. 309], together with Corollary 4.2 above. The details of the argument are given by Porter in [20] (where, of course, the conclusion of Corollary 4.2 is assumed). For the reader's convenience we quote them.

We use the notation of [3]. Let $Z_{n}=\operatorname{Tot}_{n} \Pi^{*}\left\{Y_{\alpha}\right\}$, with the natural base point. There is a pointed tower of fibrations $\left\{Z_{n}\right\}$ whose simplicial inverse limit is $Z \equiv \operatorname{holim}\left\{Y_{\alpha}\right\}$. The spectral sequence to be used is that associated

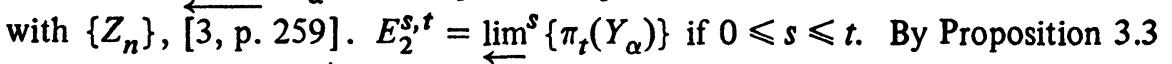
and Corollary $4.2, E_{2}^{s, t}=0$ unless $s=0$. The differential has bi-degree $(r, r-1)$, so $E_{2}^{s, t} \cong E_{r}^{s, t} \cong E_{\infty}^{s, t}$, for all $r$.

The case $i=0$ is treated by the Connectivity Lemma, p. 261 of [3], from which it follows that $Z \equiv \operatorname{holim}\left\{Y_{\alpha}\right\}$ is connected. 
From now on, we assume $i \geqslant 1$. By Adams' Lemma, p. 263 of [3], the spectral sequence is completely convergent. Hence the natural homomorphisms

$$
\pi_{i}\left(\operatorname{holim}\left\{Y_{\alpha}\right\}\right) \equiv \pi_{i}(Z) \rightarrow \underset{\lim }{\longleftarrow}\left\{\pi_{i}\left(Z_{n}\right)\right\}
$$

and

$$
e_{\infty}^{s, s+i} \rightarrow E_{\infty}^{s, s+i} \rightarrow E_{2}^{s, s+i}
$$

are isomorphisms. Thus the natural homomorphism

$$
Q_{s} \pi_{i}(Z) \rightarrow Q_{s-1} \pi_{i}(Z), \quad s \neq 0
$$

is a monomorphism and hence is an isomorphism (since it is clearly onto); when $s=0, Q_{s-1} \pi_{i}(Z)=0$, so $Q_{0} \pi_{i}(Z) \equiv e_{\infty}^{0, i}$ is naturally isomorphic to $\lim _{\longleftarrow}\left\{\pi_{i}\left(Y_{\alpha}\right)\right\}$. But there are natural isomorphisms

$$
\stackrel{\lim }{\longleftarrow}\left\{\pi_{i}\left(Z_{n}\right)\right\} \leftarrow \lim \left\{Q_{n} \pi_{i}(Z)\right\} \rightarrow Q_{0} \pi_{i}(Z) .
$$

Combining, we find that $p$ induces an isomorphism $\pi_{i} \stackrel{\text { holim }}{\longleftarrow}\left\{Y_{\alpha}\right\} \rightarrow$ $\lim _{\longleftarrow}\left\{\pi_{i}\left(Y_{\alpha}\right)\right\}$.

Next we recall some well-known facts about mapping cylinders. If $f_{\alpha}: X_{\alpha}$ $\rightarrow Y_{\alpha}$ is a morphism of $T_{0}$, the following diagram commutes in $T_{0}$ :

$\left(*_{\alpha}\right)$

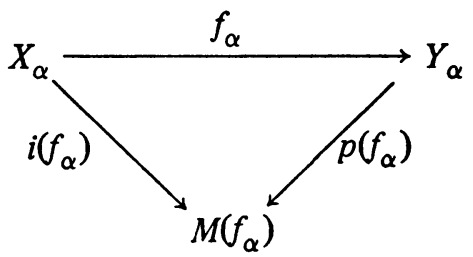

where $M\left(f_{\alpha}\right)$ is the reduced mapping cylinder, $i\left(f_{\alpha}\right)$ is the natural inclusion and $p\left(f_{\alpha}\right)$ is the natural projection map. $p\left(f_{\alpha}\right)$ is a pointed homotopy equivalence. If the following diagram in $T_{0}$ commutes on passing to $H T_{0}$

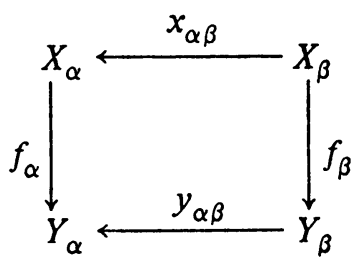

then, in order to get an induced morphism $\left(*_{\beta}\right) \rightarrow\left(*_{\alpha}\right)$ in $H T_{0, \Delta}$, one must choose a pointed homotopy $F_{\alpha \beta}: X_{\beta} \times I \rightarrow Y_{\alpha}$ between $f_{\alpha}{ }^{\circ} x_{\alpha \beta}$ and $y_{\alpha \beta}{ }^{\circ} f_{\beta}$. Define $m_{\alpha \beta}: M\left(f_{\beta}\right) \rightarrow M\left(f_{\alpha}\right)$ by $m_{\alpha \beta}([x, t])=\left[x_{\alpha \beta}(x), 2 t\right]$ if $0 \leqslant t \leqslant 1 / 2$, $m_{\alpha \beta}([x, t])=F_{\alpha \beta}(x, 2 t-1)$ if $1 / 2 \leqslant t \leqslant 1$. The maps $x_{\alpha \beta}, y_{\alpha \beta}$ and $m_{\alpha \beta}$ then induce a morphism of $H T_{0, \Delta}$ as required.

Now, suppose that in the following diagram in $T_{0}$, the triangles commute 
in $T_{0}$ while the squares commute on passing to $H T_{0}$.

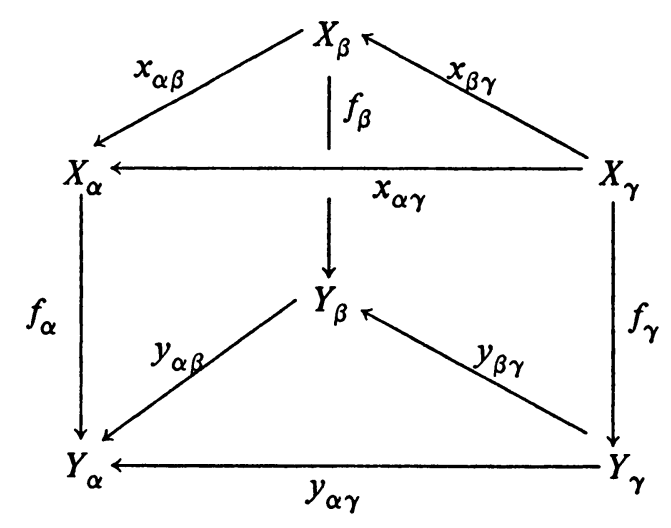

Let $F_{\alpha \beta}, F_{\beta \gamma}$ and $F_{\alpha \gamma}$ be pointed homotopies making the squares commute, and let $m_{\alpha \beta}, m_{\beta \gamma}$ and $m_{\alpha \gamma}$ be the corresponding maps between the mapping cylinders. Then there is an induced diagram in $H T_{0, \Delta}$ :

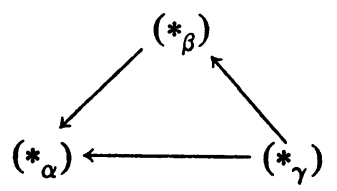

This last diagram will commute in $H T_{0, \Delta}$ provided the homotopies $F_{\alpha \beta}, F_{\beta \gamma}$ and $F_{\alpha \gamma}$ are "coherent", i.e. provided there is a "higher" pointed homotopy $F_{\alpha \beta \gamma}$ : $X_{\gamma} \times \Delta \rightarrow Y_{\alpha}$ where $\Delta$ is a standard 2-simplex, which agrees with $F_{\alpha \gamma}, F_{\alpha \beta}$ 。 $\left(x_{\beta \gamma} \times 1\right)$ and $y_{\alpha \beta} \circ F_{\beta \gamma}$ on the appropriate faces of $\Delta$.

If $\left\{X_{\alpha}\right\}$ and $\left\{Y_{\alpha}\right\}$ are objects of $\left(T_{0}\right)^{A}$, a morphism $\left\{X_{\alpha} \stackrel{f_{\alpha}}{\longrightarrow} Y_{\alpha}\right\}$ of $\left(H T_{0}\right)^{A}$ will be called coherent if for every $\alpha \leqslant \beta$ there is $F_{\alpha \beta}: X_{\beta} \times I \rightarrow Y_{\alpha}$, and for every $\alpha \leqslant \beta \leqslant \gamma$ there is $F_{\alpha \beta \gamma}: X_{\gamma} \times \Delta \rightarrow Y_{\alpha}$, as above. We have proved:

LEMma 5.2. With notation as above, if $f \equiv\left\{X_{\alpha} \stackrel{f_{\alpha}}{\longrightarrow} Y_{\alpha}\right\}$ is coherent, then the following diagram commutes in $\left(H T_{0, \Delta}\right)^{A}$

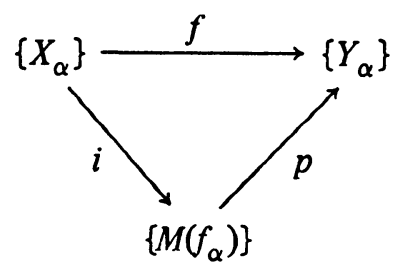

and $p$ is invertible.

We can now state the appropriate Whitehead Theorem: 
THEOREM 5.3. Let $\left\{X_{\alpha}\right\}$ and $\left\{Y_{\alpha}\right\}$ be objects of $\left(C W_{0}\right)^{A}$ of finite $C W$ dimension. Let $f \equiv\left\{X_{\alpha} \stackrel{f_{\alpha}}{\longrightarrow} Y_{\alpha}\right\}$ be a coherent morphism of $\left(H_{0}\right)^{A}$ such that for all $i \geqslant 1,\left\{\pi_{i}\left(X_{\alpha}\right) \stackrel{f_{\alpha \#}}{\longrightarrow} \pi_{i}\left(Y_{\alpha}\right)\right\}$ induces an isomorphism in pro-Groups. Then $f$ induces an isomorphism in pro- $H_{0}$.

Proof. This follows from Lemma 5.2 together with the proof of the Whitehead Theorem in $\S 3$ of [5] (Lemma 5.2 allows one to "enter" that proof at Lemma 3.7 of [5]).

The main theorem of this section is

TheOREM 5.4. Let $X \equiv\left\{X_{\alpha}\right\}_{\alpha \in A}$ be an object of pro-CW .

(i) There exists a pointed connected $C W$ complex $Q$ and a weak equivalence $q: Q \rightarrow X$ in pro- $H_{0}$ if and only if $\left\{\pi_{i}\left(X_{\alpha}\right)\right\}$ is dominated in pro-Groups by a group, for each $i \geqslant 1$. In case the condition in (i) holds $Q$ and $q$ may be chosen so that:

(ii) $C W-\operatorname{dim} Q=\max \{3, h-\operatorname{dim} X\}$, and if $h-\operatorname{dim} X=1, Q$ can be a bouquet of circles;

(iii) if s-h-dim $X<\infty, q$ induces an isomorphism in pro- $H_{0}$;

(iv) if $s-h-\operatorname{dim} X<\infty$, and $X$ is compact then $Q$ is dominated (in $H_{0}$ ) by a finite complex.

Proof OF (i). By Lemma 5.1, the required $Q$ is $\mid$ holim $\left\{S\left(X_{\alpha}\right)\right\} \mid$, and $q$ is the composition

$$
Q \stackrel{|p|}{\longrightarrow}\left\{\left|S\left(X_{\alpha}\right)\right|\right\} \stackrel{\psi}{\longrightarrow}\left\{X_{\alpha}\right\}
$$

where $\psi$ is the isomorphism of pro- $H_{0}$ induced by the canonical maps $\psi_{\alpha}$ : $\left|S\left(X_{\alpha}\right)\right| \rightarrow X_{\alpha}$.

Proof OF (ii). Let $q: Q \rightarrow X$ be as in (i). The argument used in the proof of Theorem 4.2(ii) of [5] (which is based on Theorems D and E of [22]) shows that $Q$ is pointed homotopy equivalent to a complex $Q^{*}$ with the required properties. If $q^{*}: Q^{*} \rightarrow Q$ is a pointed homotopy equivalence then $q^{\circ} q^{*}: Q^{*}$ $\rightarrow X$ is a weak equivalence in pro- $H_{0}$.

Proof of (iii). We may assume $C W$-dim $X<\infty . p$ is defined by means of maps

$$
p_{\alpha_{0}}: \underset{\operatorname{holim}}{\longleftarrow}\left\{S\left(X_{\alpha}\right)\right\} \rightarrow S\left(X_{\alpha_{0}}\right) .
$$

Let $\left\{Q_{\alpha}\right\}_{\alpha \in A}$ be the constant system defined by $Q$, i.e. $Q_{\alpha}=Q$ for all $\alpha$, and all bonds are identity maps. By applying Proposition 3.4, p. 296, of [3], and then taking geometric realizations, we note that collection of maps 
$\left\{Q_{\alpha} \stackrel{|p|}{\longrightarrow} S\left(X_{\alpha}\right)\right\}$ induces a coherent morphism of $\left(H_{0}\right)^{A}$. Now $\left\{\left|S\left(X_{\alpha}\right)\right| \stackrel{\psi_{\alpha}}{\longrightarrow}\right.$ $\left.X_{\alpha}\right\}$ is a morphism of $\left(C W_{0}\right)^{A}$, as is $\left\{Q_{\alpha}^{*} \stackrel{q^{*}}{\longrightarrow} Q_{\alpha}\right\}$. Hence the collection

$$
\left\{Q_{\alpha}^{*} \stackrel{q^{*}}{\longrightarrow} Q_{\alpha} \stackrel{\left|p_{\alpha}\right|}{\longrightarrow} S\left(X_{\alpha}\right) \stackrel{\psi}{\longrightarrow} X_{\alpha}\right\}
$$

induces a coherent morphism of $\left(H_{0}\right)^{A}$ between objects $\left\{Q_{\alpha}^{*}\right\}$ and $\left\{X_{\alpha}\right\}$ of finite $C W$ dimension. By Theorem 5.3, the resulting morphism of pro- $H_{0}$ is invertible.

Proof of (iv). Similar to the proof of 4.2(iv) in [5]. One needs $s-h$-dim $X<\infty$ to use (iii); as in [5], one needs the fact that $\lim _{\longleftarrow}\left\{X_{\alpha}\right\}$ is a compact space.

REMARK 5.5. Theorem 5.4(iv) should be read in conjunction with Remark 3.7.

REMARK 5.6. In the spirit of Remark 3.8, we conjecture that, for an object $X$ of pro- $C W_{0}$, there exist a tower $Q$ in pro- $C W_{0}$ and a weak equivalence $q$ : $Q \rightarrow X$ if and only if each $\pi_{k}(X)$ is dominated in pro-Groups by a tower in proGroups.

6. Algebraic criteria for stability in shape. A pointed connected space $Z$ has strong shape dimension [resp. shape dimension] $\leqslant n$ if there is an object $X$ of pro- $C W_{0}$ [resp. pro- $H_{0}$ ] associated with $Z$ such that $C W-\operatorname{dim} X \leqslant n$. Although we shall not use the fact, it is worth noting that there is an object of pro- $C W_{0}$ associated with every topological space $Z$ : one applies the Vietoris functor [19] based on locally finite open normal covers of $Z$ exactly one of whose elements contains the base point [18], together with [4].

So that our Theorem 6.3 may be relevant, we prove

Proposition 6.1. If a (pointed connected) separable metric space $Z$ has covering dimension $\leqslant n$, then $Z$ has strong shape dimension $\leqslant 2 n+1$.

Proof. Embed $Z$ in euclidean $(2 n+1)$-space [9]. The system of all connected open neighborhoods of $Z$, pointed by the base point of $Z$ and bonded by inclusions, is an object of pro-CW $W_{0}$. Even if $Z$ is not closed, this object is associated with $Z$ in the sense of Fox [8], see [10], and hence [18, Theorem 2.5] the induced object of pro- $H_{0}$ is associated with $Z$. $\square$

REMARK 6.2. An $n$-dimensional compact metric space, being the inverse limit of nerves of covers, has strong shape dimension $n$.

Here is our stability theorem:

THEOREM 6.3. Let $Z$ be a pointed connected space whose strong shape dimension is finite. Then $Z$ is pointed shape equivalent to a $C W$ complex if and 
only if each pro- $\pi_{k}(Z)$ is dominated in pro-Groups by a group. This complex may be chosen to have $C W$-dimension $\max \{3$, shape dimension of $Z\}$, and to be a bouquet of circles if the shape dimension of $Z$ is 1 . If, in addition, $Z$ is compact, then $Z$ is pointed shape dominated by a finite complex. In particular, the theorem holds when $Z$ is a finite-dimensional separable metric space.

Proof. Immediate from Theorem 5.4 and Proposition 6.1.

REMARK 6.4. When $Z$ is compact, the theorem should be read in conjunction with Remark 3.7.

Note (added November 1975). Since this paper was submitted, a Whitehead Theorem in pro- $H_{0}$ more general than Theorem 5.3 has appeared, due to Morita [23]. As a result, Theorem 6.3 now holds for spaces of finite shape dimension.

\section{REFERENCES}

1. M. Artin and B. Mazur, Etale homotopy, Lecture Notes in Math., no. 100, Springer-Verlag, Berlin and New York, 1969. MR 39 \#6883.

2. K. Borsuk, Concerning homotopy properties of compacta, Fund. Math. 62 (1968), 223-254. MR 37 \#4811.

3. A. K. Bousfield and D. M. Kan, Homotopy limits, completions and localizations, Lecture Notes in Math.,vol. 304, Springer-Verlag, Berlin and New York, 1973.

4. C. H. Dowker, Homology groups of relations, Ann. of Math. (2) 56 (1952), 8495. MR 13, 967.

5. D. A. Edwards and R. Geoghegan, The stability problem in shape and a Whitehead theorem in pro-homotopy, Trans. Amer. Math. Soc. 214 (1975), $261-277$.

6. Shapes of complexes, ends of manifolds, homotopy limits and the Wall obstruction, Ann. Math. 10 (1975), 521-535.

7. D. A. Edwards and H. Hastings, Čech and Steenrod homotopy theories with applications to geometric topolog.y. Lect ure Notes in Math., Springer-Verlag, Berlin and New York (to appear).

8. R. H. Fox, On shape, Fund. Math. 74 (1972), no. 1, 47-71. MR 45 \#5973; erratum, 46 \#6282.

9. W. Hurewicz and H. Wallman, Dimension theory, Princeton Math. Ser., vol. 4, Princeton Univ. Press, Princeton, N. J., 1941. MR 3, 312.

10. D. M. Hyman, A remark on Fox's paper on shape, Fund. Math. 75 (1972), no. 3, 205-208. MR 46 \#2635.

11. G. Kozlowski and J. Segal, Locally well-behaved paracompacta in shape theory, Fund. Math. (to appear).

12. J. Levan, Doctoral dissertation, Univ. of Kentucky, 1973.

13. S. Mardešić, Shapes for topological spaces, General Topology and Appl. 3 (1973), 265-282. MR 48 \#2988.

14. - On the Whitehead theorem in shape theory, Fund. Math. (to appear).

15. S. Mardešić and J. Segal, Shapes of compacta and ANR-systems, Fund. Math. 72 (1971), no. 1, 41-59. MR 45 \#7686.

16. Equivalence of the Borsuk and the ANR system approach to shapes, Fund. Math. 72 (1971), no. 1, 61-68. MR 46 \#850.

17. J. P. May, Simplicial objects in algebraic topology, Math. Studies, no. 11, Van Nostrand, Princeton, N. J., 1967. MR 36 \#5942.

18. K. Morita, On shapes of topological spaces, Fund. Math. 86 (1975), 251-259.

19. T. Porter, Čech homotopy. I, II, J. London Math. Soc. (2) 6 (1973), 429-436, 667-675. MR 47 \#613; 50 \#8517a.

20. Stability results for topological spaces, Math. Z. 140 (1974), 1-21. 
21. J.-L. Verdier, Équivalence essentielle des systèmes projectifs, C. R. Acad. Sci. Paris 261 (1965), 4950-4953. MR 32 \#7623.

22. C. T. C. Wall, Finiteness conditions for CWcomplexes, Ann. of Math. (2) 81 (1965), 55-69. MR 30 \#1515.

23. K. Morita, The Hurewicz and the Whitehead Theorems in shape theory, Sci. Rep. Tokyo Kyoiku Daigaku Sect. A 12 (1974), 246-258.

DEPARTMENT OF MATHEMATICS, STATE UNIVERSITY OF NEW YORK, BING. HAMTON, NEW YORK 13901 